\title{
B-Spline Surface Modelling with Adaptive de Boor Grids in Hydroinformatics
}

\author{
C. Lichy ${ }^{1}$ and V. Berkhahn ${ }^{2}$ \\ 1) Franzius-Institute for Hydraulic, Waterways and Coastal Engineering, University of \\ Hannover, Nienburger Str.4, 30167 Hannover, Germany,Email: cl@fi.uni-hannover.de, \\ 2) Institute for Computer Science in Civil Engineering, University of Hannover, Am Kleinen \\ Felde 30,30167 Hannover, Germany Email: berkhahn@bauinf.uni-hannover.de
}

Key words: Surface Modelling, B-Splines, Adaptive de Boor Grids

Abstract: A high quality surface representation is indispensable for a hydrodynamic numerical model, which is related to nature. Standard algorithms for meshing are not able to guarantee these requirements. Applied methods approximate the surface by patches. As a result an implementation for a b-spline surface was realised and tested. The used method ,tensor product b-spline surface“ is defined by an array of control points, called de Boor points. The associated bspline functions are defined in every local segment. Changes at one point of the control point mesh result only in local changes of the b-spline surface.

\section{INTRODUCTION}

To provide a sufficient hydrodynamic model in coastal engineering a high quality representation of the bathymetry and the topography of the coast line is required. The geometric description of the bathymetry is based on measurements, and include a number of uncertainties. These are the usual errors in measuring, but also overlapping and blanking of measurement areas. For the description of the surface, methods are required that offer a unique and gapless information on the geometric charactertics of the natural surface.

Furthermore discontinuities in the bathymetry, like break lines, are an additional problem in coastal engineering. Because for a point on the surface, with preselected $\mathrm{x}$ - and $\mathrm{y}$ - coordinates, multiple $\mathrm{z}$ - coordinates can

The original version of this chapter was revised: The copyright line was incorrect. This has been corrected. The Erratum to this chapter is available at DOI: 10.1007/978-0-387-35503-0_29 
not be assigned, the geometric description and also the automatic mesh generation is complex. The presented methods for the description of the surface are tested for their suitability in coastal engineering. The mathematical basics for the description of free form geometry are presented first for curves, because of the more vivid description. At next the necessary equations are developed for surfaces.

\section{BÉZIER CURVES}

The algorithm described in this section is probably the most fundamental one in the field of curve and surface design, yet is surprisingly simple. Its main attraction is the beautiful interplay between geometry and algebra: a very intuitive geometric construction leads to a powerful theory.Bézier curves can be defined by a recursive algorithm, which de Casteljau developed at first in 1959 for the geometric construction of free form curves (de Casteljau, 1959). However, it is also necessary to have an explicit representation of the curves, i.e. to express a non recursive formula rather in terms of a recursive algorithm. This will facilitate further theoretical development considerably.

The curve $\mathbf{b}(t)$ is given by the parameter $t$ to get a description for any free form curve. This means, the coordinates of a curve point $\mathbf{b}(t)$ are independent functions of the curve parameter $t$. The parameter interval is usually normalised into the interval 0.0 to 1.0 .

$$
\mathbf{b}(t)=[x(t), y(t), z(t)]^{T} \text { with } \mathbf{x} \in \boldsymbol{E}^{3}, t \in[0.0,1.0]
$$

According to the de Casteljau algorithm, Bézier curves are presented as linear, recursive interpolation with the curve parameter $t$ between the control points $\mathbf{b}_{\mathrm{i}}$ or between the control points $\mathbf{b}_{i}^{r-1}$ of the $\mathrm{r}^{\text {th }}$ recursion step (Farin, 1993, Hoschek \& Lasser, 1992)

$$
\mathbf{b}_{i}^{r}(t)=(1-t) \mathbf{b}_{i}^{r-1}(t)+t \mathbf{b}_{i+1}^{r-1}(t) \text { with } \mathbf{b}_{i}^{0}=\mathbf{b}_{i} .
$$

If a Bézier curve is described by $n+1$ control points $\mathrm{b}_{0}, \ldots, \mathrm{b}_{n}$, then the control point $\mathbf{b}_{i}^{n}$ of the $n^{\text {th }}$ recursion step can be found on the curve. The control points $b_{0}$ and $b_{n}$ are the endpoints of the Bézier curve. The directions of the tangents of these points are defined by the start and end edges of the control polygon $\left(b_{1}-b_{0}\right)$ and $\left(b_{n}-b_{n-1}\right)$. Bézier curves will be expressed in terms of Bernstein polynomials, as explicitly defined by: 
$\mathbf{b}(t)=\sum_{i=0}^{n} \mathbf{b}_{i} B_{i}^{n}(t)$ with $\mathbf{b}_{0}, \ldots, \mathbf{b}_{n} \in \boldsymbol{E}^{3}$ and $B_{i}^{n}(t)=\left(\begin{array}{c}n \\ i\end{array}\right) t^{i}(1-t)^{n-i}$ for $i=0, \ldots n$.

The binomial coefficients are given by:

$$
\left(\begin{array}{l}
n \\
i
\end{array}\right)=\left\{\begin{array}{cc}
\frac{n !}{i !(n-i) !} & \text { if } 0 \leq i \leq n \\
0 & \text { else. }
\end{array}\right.
$$

The intermediate de Casteljau points can be expressed in terms of Bernstein polynomials of degree $r$ :

$$
\mathbf{b}_{i}^{r}(t)=\sum_{j=0}^{r} \mathbf{b}_{i+j} B_{i}^{r}(t) \text { with } r \in\{0, \ldots, n\}, i \in\{0, \ldots, n-r\}
$$

\section{B-SPLINE CURVES}

To get the flexibility needed for the modelling of a bathymetry for the curve, respectively for the surface, a number of Bézier curve segments of the same degree are connected (Farin, 1993, Hoschek \& Lasser, 1992). Two Bézier functions defined by $\left[u_{\mathrm{i}-1}, u_{\mathrm{i}}\right]$ and $\left[u_{\mathrm{i}}, u_{\mathrm{i}+1}\right]$ are $\mathrm{C}^{2}$ continuity at $u_{\mathrm{i}}$ if an auxiliary point $d_{i}$ can be constructed of both curve segments.

$$
\begin{aligned}
& \mathbf{d}_{i-}=\frac{u_{i+1}-u_{i-1}}{u_{i}-u_{i-1}} \mathbf{b}_{i-1}-\frac{u_{i+1}-u_{i}}{u_{i}-u_{i-1}} \mathbf{b}_{i-2} \\
& \mathbf{d}_{i+}=\frac{u_{i+1}-u_{i-1}}{u_{i+1}-u_{i}} \mathbf{b}_{i+1}-\frac{u_{i}-u_{i-1}}{u_{i+1}-u_{i}} \mathbf{b}_{i+2} \\
& \mathbf{d}_{i}=\mathbf{d}_{i-}=\mathbf{d}_{i+}
\end{aligned}
$$

Therefore, the control points near every connection of the curve segments can not be freely chosen. A curve notation where only the freely choosen control points are taken into consideration is called basis spline curve or b- 
spline curve. These control points are named de Boor points. With $N$ as the number of segments and $K$ as the degree of the b-spline curve the knot vector $u$ with the global knot parameters $u_{i}$ is defined as:

$$
\mathbf{u}=\left[u_{0}, \ldots, u_{N+k+1}\right]^{T} \text { with } N, K \in N ; N \geq K \geq 1 ; u_{i} \in \boldsymbol{R}
$$

The local parameter $t_{i}$ for the $\mathrm{i}^{\text {th }}$ curve segment is calculated by the linear interpolation between the knot parameters $u_{\mathrm{i}}$ and $u_{\mathrm{i}+1}$. The starting b-spline functions are piecewise constant in every segment:

$$
N_{i}^{0}(u)=\left\{\begin{array}{l}
1.0 \text { for } u \in\left[u_{i}, u_{i+1}[\quad \text { for } i=0, \ldots, N\right. \\
0.0 \text { else }
\end{array}\right.
$$

According to the b-spline function of the $(r-1)^{\text {th }}$ recursion step the bspline function of the $r^{\text {th }}$ recursion step is given by:

$$
N_{i}^{r}(u)=\frac{u-u_{i}}{u_{i+r}-u_{i}} N_{i}^{r-1}(u)+\frac{u_{i+r+1}-u}{u_{i+r+1}-u_{i+1}} N_{i+1}^{r-1}(u)
$$

$$
\text { for } r=1, \ldots, N+K ; i=0, \ldots, N+K-r \text {. }
$$

b-spline functions only have a value different from zero in the open interval $u \in] u_{\mathrm{i}}, u_{\mathrm{i}+\mathrm{K}+1}[$. Outside this interval the value is always equal to zero. This indicates that with a given global parameter $u \in\left[u_{i}, u_{i+1}\right]$ only the b-spline functions $N_{i-K}^{K}(u), \ldots, N_{i}^{K}(u)$ have a value larger than zero, and only the control points $d_{i-K}, \ldots, d_{i}$ influence the shape of the curve. In this way, local modelling with $b$-spline curves is possible.

The b-spline curve depending on the global curve parameter $u$ with the knot vector $\mathbf{u}$, the b-spline functions $N_{i}^{K}$ and the de Boor points $\mathbf{d}_{\mathrm{i}}$ is given by:

$$
\mathbf{b}(u)=\sum_{i=0}^{N} \mathbf{d}_{i} N_{i}^{K}(u)
$$

with $N, K \in N ; N \geq K \geq 1 ; \mathbf{d}_{0}, \ldots, \mathbf{d}_{N} \in \boldsymbol{E}^{3} ; u \in\left[u_{K}, u_{N+1}\right]$. 
The only method to build the sum of (control) points is the affine combination, where the sum of all factors of the (control) points is equal to 1. In the intervals $\left[u_{0}, u_{K}[\right.$ and $\left.] u_{N+1}, u_{N+K+1}\right]$ the sum of the b-spline function is not equal to 1 and therefore no affine combination of the control points is possible. For b-spline curves the definition of the global parameter $u$ is limited to the interval $\left[u_{\mathrm{K}}, u_{\mathrm{N}+1}\right]$, since only in this interval the sum of the bspline functions is equal to 1 . The knot parameter $u_{0}$ and $u_{N+K+1}$ have no influence on the b-spline functions in the concidered interval $\left[u_{K}, u_{N+1}\right]$ of the b-spline curve. Therefore, the first and last knot parameter are choosen as $u_{0}$ $=u_{1}$ and $u_{N+K+1}=u_{N+K}$. Generally, there is no end point interpolation for the b-spline curve at the start and end control points $\mathbf{d}_{0}$ and $\mathbf{d}_{N}$. Obviously this behaviour causes problems during the process of modelling. This problem is solved by using a special knot vector $\mathbf{u}$. The shapes of Bézier curves and bspline curves are controlled by points $\mathbf{b}_{i}$ respectively de Boor points $\mathbf{d}_{i}$. bspline curves, in contrast to Bézier curves, influence the shape of the curve via the knot vector $\mathbf{u}$. K multiple knots $u_{j+1}=\ldots=u_{j+K}=u^{*}$ effect:

$$
\mathbf{d}_{j}=\mathbf{b}\left(u^{*}\right) \text { for } j=0, \ldots, N
$$

The de Boor point $\mathbf{d}_{j}$ lies on the curve and the tangents next to $\mathbf{d}_{j}$ are the edges $\mathbf{d}_{j}-\mathbf{d}_{j-1}$ and $\mathbf{d}_{j+1}-\mathbf{d}_{j}$ of the control polygon. The end point interpolation is possible for $\mathrm{K}$ multiple knots $\mathrm{u}_{1}=\ldots=u_{K}$ and $u_{N+1}=\ldots=u_{N+K}$. In the special case of $u_{1}=\ldots=u_{\mathrm{k}}=0.0$ and $u_{N+1}=\ldots=u_{N+K}=1.0$ the b-spline functions $N_{i}^{K}(u)$ are equal to the Bernstein polynomials $B_{i}^{n}(t)$, with $n=K$ and $i=0, \ldots, n$.

\section{B-SPLINE SURFACES}

B-spline surfaces play an important role in current surface design methods and will be discussed here in more detail. Using the notation made in section 3 , a parametric tensor product b-spline surface may be written as

$$
\mathbf{b}(u, v)=\sum_{\mathrm{i}=0}^{\mathrm{N}} \sum_{\mathrm{j}=0}^{\mathrm{M}} \mathbf{d}_{\mathrm{ij}} N_{i}^{K}(u) N_{j}^{L}(v)
$$

$$
\begin{aligned}
& \text { with } N, M, K, L \in N ; N \geq K \geq 1 ; M \geq L \geq 1 ; \\
& \mathbf{d}_{00}, \ldots, \mathbf{d}_{N M} \in E^{3} ; u \in\left[u_{K}, u_{N+1}\right] ; v \in\left[v_{L}, v_{M+1}\right] .
\end{aligned}
$$


It is assumed that one knot vector $\mathbf{u}$ in the $u$-direction and one knot vector $\mathbf{v}$ in the $v$-direction is given. Bézier surfaces and $b$-spline surfaces have a number of advantages compared with the common methods for surface description. Two of the most important advantages are: Variation diminishing property: The surface is not intersected by any straight line more often than is the control grid. An oscillation of the surface is impossible. Strong convex hull property: Each point on the surface lies in the convex hull of more than $n+1$ nearby control points. This property leads to an effectiv estimate and control for the location of the Bézier surfaces and bspline surfaces.

\section{MODELLING OF BATHYMETRY}

The recording of the bathymetry is done by sounding the bottom of the sea. Therefore, the water depth is measured in intervals and the position of the points of measurement is noticed. Afterwards the values are related to the reference value and stored in the order of measurement. For the modelling of larger areas the data of the patches are simply added. To connect the coastal line data sets of land surveying are also added. Because of the different measure and store techniques the start up representation is an unstructured cloud of points. The job is to approximate an b-spline surface on this unstructured cloud of points. Therefore, the position of every point $\mathbf{p}_{i}$ on the surface has to be determined:

$$
\mathbf{p}_{i}=\mathbf{b}(u, v)
$$

This leads to an over determined system of equations:

$$
\mathbf{A}_{(n, m)} * \mathbf{x}_{(m, l)}=\mathbf{b}_{(n, l)}
$$

With $n>m$ and $\operatorname{rank}(\mathbf{A})=n$. This system can be solved by using the householder transformation.

\section{EXAMPLE}

Figure 1 shows the digitised points of a part of a tidal harbour. In this example the process of approximation starts up with more than 8.000 points. The result of the approximation is a single surface controlled a by $50 \times 50$ point de Boor grid. The equidistant de Boor point grid is shown in figure 2. 


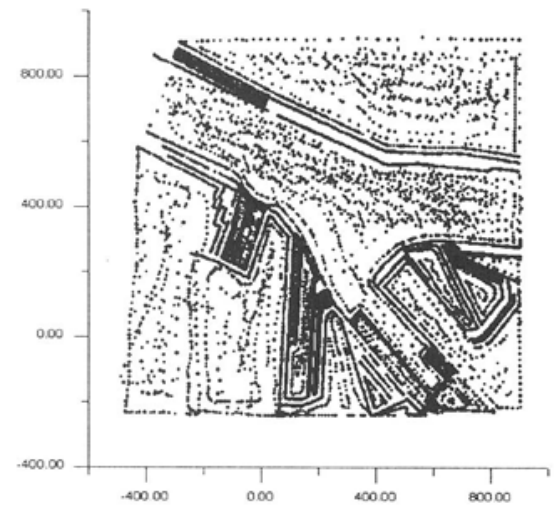

Figure 1: Digitised Points

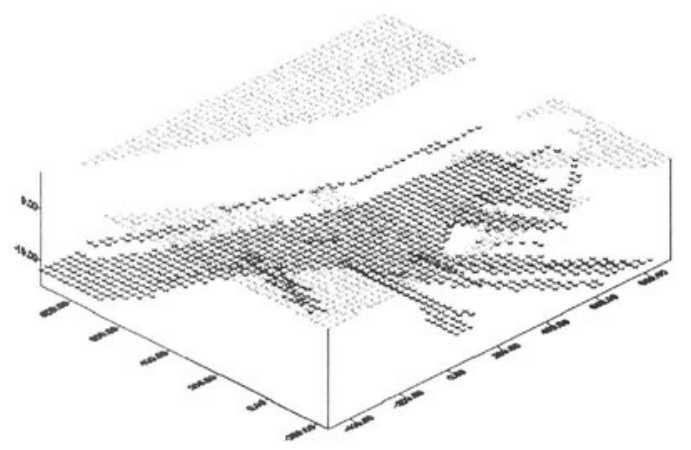

Figure 2: De Boor Point Grid

The calculated surface is based on all the given points. Because it is just one surface element and not patched from a number of elements, there are no problems at the edges. To show the quality of the representation a part of the surface plot is expanded and shown in figure 3 . 


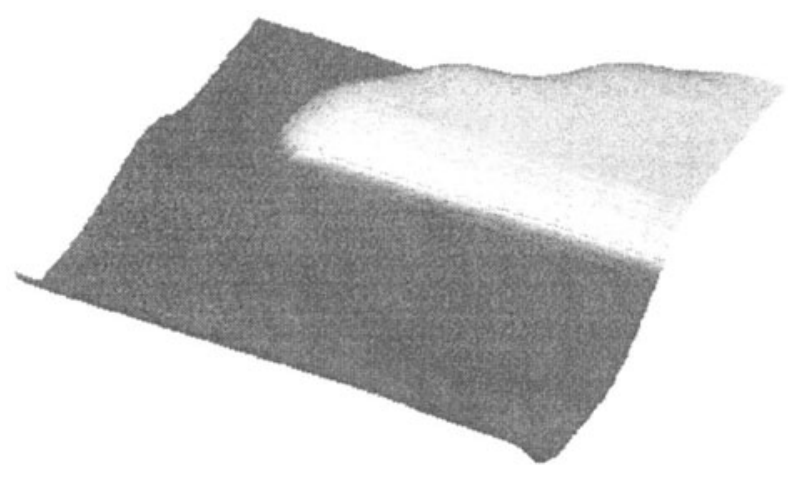

Figure 3: Detail Plot

\section{ADAPTIVE DE BOOR POINT GRIDS}

The example has illustrate, that bathymetry modelling using b-Spline surfaces can be rather simple. Break lines and blanking areas can easily be handled. Usually the approximation of a relief is done by a number of patches. The problem is to define the topological connections between these patches. The presented area is described by a single b-Spline surface. Especially the detail shown in figure 4 demonstrates the disadvantage of a bspline surface representation with an equidistant de Boor point grid. The approximation of break line, which are not parallel to the de Boor point grid, leads to an unacceptable deviation of the surface with respect to the break lines and has to be improved. A very simple idea to improve the approximation is the insertion of additional de Boor points in the areas of unacceptable surface deviation. The de Boor point grid has to be regular; this means that the control points consist of $N$ columns and $M$ rows. If an additional control point should be inserted to improve the approximation, an additonal control point has to be inserted in every of the $N$ columns and $M$ rows. This leads to great numerical effort if serveral additional points are to be inserted. Therefore, a method to improve the approximation quality without increasing the number of control points is presented. Instead of inserting points, the control points are moved in the $u-v$-parameter plane for a better fit of break lines. In areas with large changes in the surface tangents the density of de Boor points is increased. On the other hand, in area with small changes in the surface tangents the density of the control points is decreased. This method leads to an adaptive de Boor point grid.

At the first iteration step the surface approximation is based on an equidistant de Boor point grid.

At first iteration step the surface approximation is based on an equidistant de Boor point grid. For every value in the knot matrix the associated surface point $\mathrm{b}\left(u_{i}, v_{\mathrm{j}}\right)$ are calculated. The changes of the surface tangents in the point $\mathrm{b}\left(u_{i}, v_{\mathrm{j}}\right)$ are given by 


$$
\frac{\Delta \mathbf{b}\left(u_{i}, v_{j}\right)}{\Delta u_{i}}=\frac{\mathbf{b}\left(u_{i+1}, v_{j}\right)-\mathbf{b}\left(u_{i}, v_{j}\right)}{u_{i+1}-u_{i}}-\frac{\mathbf{b}\left(u_{i}, v_{j}\right)-\mathbf{b}\left(u_{i-1}, v_{j}\right)}{u_{i}-u_{i-1}}
$$

The measure of the movement of the parameter $\boldsymbol{u}_{j}$ is determined by the scaling factor $\alpha$ and

$$
\frac{\Delta \Delta \mathbf{b}\left(u_{i}, v_{j}\right)}{\Delta \Delta u_{i}}=\frac{\Delta \mathbf{b}\left(u_{i+1}, v_{j}\right)}{\Delta u_{i+1}}-\frac{\Delta \mathbf{b}\left(u_{i-1}, v_{j}\right)}{\Delta u_{i-1}}
$$

The new parameter $u_{i}^{*}$ for the adaptive de Boor point grid is

$$
u_{i}^{*}=u_{i}+\alpha \frac{\Delta \Delta \mathbf{b}\left(u_{i}, v_{j}\right)}{\Delta \Delta u_{i}}
$$

Corresponding equations can be derived for parameter $v_{j}{ }^{*}$ in the same manner. Tests with a break line with $30^{\circ}$ have demonstrated a significant improvement with one iteration.

\section{OUTLOOK}

Using b-spline surfaces with adaptive de Boor point grids offers new possibilities for dealing with numerical models in hydroinformatics. Because of the different origins of the used data, there are problems in handling break lines and blanked areas. Using applied methods indicates manual work to deal with these problems. Setting up the calculation grid for large models costs a lot of time and causes uncertainties on the quality. A b-spline surface with an adaptive de Boor point grid is build using determined algorithms without any manual work on the data. The opportunity to build up the calculation grid automaticly is one of the research targets. This will save a lot of time during the setup of a numerical model and guaranties a certain quality standard. Adaptive calculation grids can get the values of new points or moved points from the b-spline surface, which is a new quality in hydronumerical modelling.

The approximation of a b-spline surface includes a householder transformation. The number of rows in the householder matrix is the number of given points and the number of columns is the number of used de Boor points. Setting up large scale models causes the problem to deal with a very 
large matrix. Before the transformation the matrix contains a lot of zeros, but the location of the non zero elements is unstructured. After the transformation the matrix is an upper triangle matrix. Storing this matrix and solving the transformation costs a lot of performance. Looking for more efficient algorithms is one of the next research targets.

Further methods to improve the approximation quality have to be developed and evaluated of efficiency. Additionally, the use of multiple knots in the knots matrix and rational b-spline surfaces should be examined on sufficiency for modelling the bathymetry.

\section{REFERENCES}

Berkhahn, V., Lichy, C. 1998. Geometrische Modellierung im Küsteningenieurwesen; R. Mahnken (Hrsg.), Theoretische und numerische Methoden in der angewandten Mechanik mit Praxisbeispielen. Forschungs- und Seminarberichte aus dem Bereich der Mechanik der Universität Hannover. Bericht-Nr. F 98/4. Hannover.

de Casteljau, P. 1959. Outillages méthodes calcul. Technical Report: A. Citroen. Paris.

Farin, G. E. 1993. Curves and surfaces for computer aided geometric design: a practical guide. $3^{\text {rd }}$ Ed. London: Academic Press.

Hoschek, J., Lasser, D. 1992. Grundlagen der geometrischen Datenverarbeitung. Stuttgart: Teubner Verlag.

Lichy, C. 1998. A Multidirectional and Multifunctional Gateway between GIS and Hydrodynamic Models. Proc. of the Conference on Hydroinformatics'98. Copenhagen. Lichy, C., Berkhahn, V. Matheja, A. 1998. Geometric design in Hydroinformatics. Proc. of the Conference on Hydroinformatics'98. Copenhagen. 